Ophthalmologe 2021 · 118:534-535

https://doi.org/10.1007/s00347-021-01357-w

Angenommen: 15. Februar 2021

(c) Springer Medizin Verlag GmbH, ein Teil von Springer Nature 2021

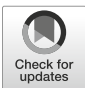

Claus Cursiefen

Zentrum für Augenheilkunde, Uniklinik Köln, Köln, Deutschland

\title{
Hochrisikokeratoplastik: die zweite (R)evolution im Hornhautbereich?
}

Das ist jedoch nur ein Teil des HornDie Therapie von Hornhauterkrankungen mittels Transplantation hat in den letzten 10 bis 15 Jahren eine „(r)evolutionäre“ Veränderung erfahren. Während in den letzten 100 Jahren fast immer unabhängig von der primären Lokalisation der Pathologie die komplette Hornhaut transplantiert wurde, hat sich dank v.a. des Siegeszugs der DMEK (Descemetmembran und Endotheltransplantation) und auch $\mathrm{zu}$ geringerem Maße der DALK (tiefe anteriore lamelläre Keratoplastik) das Feld sehr deutlich hin zu lamellären Transplantationsverfahren entwickelt [1]. Inzwischen ist weltweit, aber v. a. auch in Deutschland die DMEK der Goldstandard der Therapie bei endothelialen Erkrankungen der Hornhaut, wie der Fuchs-Endotheldystrophie, der pseudophaken bullösen Keratopathie oder auch der Pseudoexfoliationssyndrom(PEX)Keratopathie [2]. Dies liegt daran, dass die Sehschärfenerholung schneller und besser, das Immunreaktionsrisiko deutlich geringer und die intra- und postoperativen Risiken deutlich geringer sind als bei einer perforierenden Keratoplastik. Ebenso hat sich bei verschiedenen Dystrophien des vorderen Hornhautstromas, wie z. B. beim Keratokonus, die tiefe anteriore lamelläre Keratoplastik (DALK) wegen des Fehlens von endothelialen Immunreaktionen als Therapieoption etabliert. Diese Entwicklung spiegelt sich auch im Keratoplastik-Register der Sektion Kornea der Deutschen Ophthalmologischen Gesellschaft wider, wo der Anteil der lamellären Transplantationsverfahren von Jahr zu Jahr steigt und bei der letzten Umfrage schon bei über $60 \%$ lag [1]. hauttransplantationsspektrums. Es bleiben natürlich Veränderungen gerade im Bereich vaskularisierter und teilweise vernarbter oder ödematöser Hornhäute, die einer lamellären Transplantationschirurgie bisher nicht zugänglich waren und/oder mittels perforierender Keratoplastik als Hochrisikohornhaut behandelt werden müssen. Hier hat sich mit der Optimierung der Keratoprothesenchirurgie (v. a. der Boston Kpro als weltweit häufigstes durchgeführtes Verfahren) auch einiges getan. So wurde durch die telemetrische Druckmessung das Problem des Nichtmessenkönnens des Augendrucks verbessert [3]. Dennoch bleiben zahlreiche intra- und postoperative Risiken bei der Kpro-Chirurgie, die das Verfahren schlussendlich nur für einen kleineren Kreis an Patienten als Ersteingriff sinnvoll erscheinen lassen. Wir bleiben also mit einem nicht unsignifikanten Teil von Hochrisikokeratoplastiken konfrontiert, die aufgrund häufig vaskularisierter und entzündeter Empfängerbette ein deutlich erhöhtes Immunreaktionsrisiko, Wundheilungsprobleme und andere Komplikationen aufweisen.

Nun zeigen sich auch hier Anzeichen für eine deutliche mittelfristige Verbesserung der Situation durch optimierte Vorbereitung und auch neue Operationstechniken in diesen Augen. Diesen neuen Ansätzen widmet sich dieses Themenheft „Hochrisikokeratoplastik“.

Eine erste Verbesserungsmöglichkeit wurde bereits angesprochen, und zwar die Boston-Kpro-Versorgung in vaskularisierten Hochrisikoaugen, bei denen eine perforierende Keratoplastik nicht
Erfolg versprechend erscheint (z.B. bei parallel bestehender Limbusstammzelldefizienz). Hier fassen Schaub et al. [4] den aktuellen Stand der Versorgung von vaskularisierten Hochrisikoaugen mittels Boston-Kpro und auch neue Ansätze zur Augendruckmessung in diesen Augen zusammen.

Eine weitere spannende Entwicklung stellen Hos et al. dar [5]. Es geht zum einen darum, dass erste Daten darauf hinweisen, dass die DMEK auch in vaskularisierten Hochrisikoaugen mit Ödem ohne signifikante Narben zum Einsatz kommen kann. Hier führt die DMEK zu einer signifikanten Entquellung der Hornhaut und zu einer interessanten Rückbildung der pathologischen Gefäße in der Hornhaut und $\mathrm{zu}$ einem parallelen Anstieg der Sehschärfe [6]. Das Immunreaktionsrisiko ist etwas höher als bei normaler DMEK, aber immer noch deutlich geringer als bei perforierender Hochrisikokeratoplastik. Die DMEK ist also dabei, sich hier einen weiteren Bereich aus der Domäne der bisher perforierenden Hochrisikokeratoplastiken „zu erobern“. Ebenfalls ist es so, dass inzwischen gute Langzeitdaten für Sicherheit und zur Effizienz der DMEK nach Transplantatversagen nach perforierender Keratoplastik vorliegen. Auch hier sind die Daten erfreulich und zeigen, dass mittels DMEK eine schnellere und sicherere Visusrehabilitation erreicht werden kann, allerdings nur, wenn die Sehschärfenwerte nach initialer pKPL ausreichend gut waren.

Abschießend stellen Matthaei et al. eine weitere wichtige Neuentwicklung dar [7]: Es geht um die Präkonditionierung von vaskularisierten Hochrisiko- 
augen vor Transplantation. Hier weiß man aus tierexperimentellen Studien schon lange, dass die Vorbehandlung von vaskularisierten Empfängerhornhäuten vor Transplantation z.B. durch die Gefäßverödung mittels Feinnadelkauterisierung oder neuerdings auch kornealer Crosslinking-Behandlung das Transplantatüberleben bei anschließend durchgeführter Hochrisikokeratoplastik signifikant verbessert. Erste klinische Daten zeigen nun, dass dieses neue Konzept der präoperativen Lymphangioregression auch bei Patienten Erfolg hat. In einer Publikation von Hos et al. konnte gezeigt werden, dass die Vorbehandlung von vaskularisierten Hochrisikoaugen mittels Feinnadelkauterisierung und Avastin-Gabe vor anschließender Transplantation das anschließende Transplantatüberleben signifikant verbesserte [8]. Hier sind also auch mittelfristig Verbesserungen zu erwarten, die jedoch noch prospektive klinische Studien benötigen.

Zusammenfassend wünschen wir Ihnen viel Freude bei der Lektüre dieser hoffnungsvoll stimmenden neuen (r)evolutionären Ansätze auch im bisher komplexen Bereich der Hochrisikokeratoplastiken.

Mit freundlichen Grüßen aus Köln

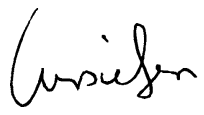

Univ.-Prof. Dr. med. C. Cursiefen Direktor des Zentrums für Augenheilkunde

\section{Korrespondenzadresse}

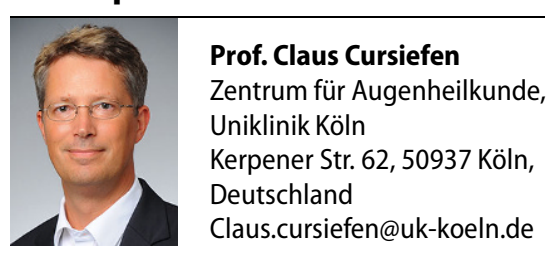

Interessenkonflikt. C. Cursiefen weist auffolgende Beziehungen hin. Beratung oder Mitarbeit in Aufsichtsräten bzw. wissenschaftlichen Beratergremien erfolgt/e bei den Firmen: Gene Signal, Schweiz; Novaliq, Heidelberg; Oxford, Biomedical, England; Mediante $\mathrm{GmbH}$, Schweiz; Medupdate, Wiesbaden; Dompe, Italien; Roche, Basel. | Wissenschaftliche Beratung erfolgt bei folgenden Zeitschriften: Der Ophthalmologe, Klinische Monatsblätter für Augenheilkunde, Graefes Archive for Clinical and Experimental Ophthalmology, Journal of Refractive Surgery, Journal of Cataract and Refractive Surgery, British Journal of Ophthalmology, Acta Ophthalmologica Scandinavica, Ophthalmologica Basel, The Lancet, Ophthalmology, Experimental Eye Research, Eye, Current Eye Research, Ophthalmic Research, PLOS, IOVS, Nature Medicine, Progress Retina Eye Research, Cornea.| Begutachtung erfolgt für folgende Einrichtungen: Deutsche Forschungsgemein schaft (DFG), Programmkommission der Deutschen Ophthalmologischen Gesellschaft (DOG), Belgischer Wissenschaftsfonds, Zentrum Molekulare Medizin Köln (ZMMK). |EBM Mitglied bei: Cornea, Der Ophthalmologe, Klinische Monatsblätter für Augenheilkunde, IOVS, Zeitschrift für Praktische Augenheilkunde.| Referent/Vortragstätigkeit mit Honorar: Alcon, Allergan, Santen, Ursapharm, Bayer, Med.-Update GmbH, Bausch\&Lomb, Haag Streit Surgical, Novaliq, Zeiss, Dompe, Heidelberg-Engeneering.|Forschungsförderung erfolgt für/durch die Firmen: Alcon, Allergan, Ursapharm, Bayer, Ruck, Bausch\&Lomb, Haag Streit Surgical, OPMEDT, Novaliq, Zeiss. DFG, EU, BMBF, Stiftungen, Roche.|Geschäftsanteile/Aktien Antragsteller/Familie: Mischfonds, Aktien. |Unentgeltliche Mitarbeit in folgenden Gremien: Visiting Professor/ Adjunct Associate Scientist, Harvard Medical School, Schepens Eye Research Institute, Boston, USA; Visiting Professor, Tongji University Shanghai, China; Vorstand, Sektion Cornea der Deutschen Ophthalmologischen Gesellschaft (DOG), München; Mitglied, Gesamtpräsidium der Deutschen Ophthalmologischen Gesellschaft (DOG), München; Mitglied, Geschäftsführendes Präsidium der Deutschen Ophthalmologischen Gesellschaft (DOG), München; Mitglied, Nationale Akademie der Wissenschaften Leopoldina, Halle; Fellow, ARVO (The Association for Research in Vision and Ophthalmology), Bethesda, USA; Fachkollegiat Ophthalmologie der Deutschen Forschungsgemeinschaft DFG, Bonn; Mitglied, Senat der Universität zu Köln, Köln; Mitglied, CIO der Universitäten Köln und Bonn; Mitglied, Geschäftsführender Vorstand, Zentrum für Molekulare Medizin der Universität Köln (CMMC), Köln; Bundesärztekammer (BÄK), Berlin, Mitglied Arbeitskreis Augenhornhautbanken; Vorsitzender, Streilein Foundation Europe, Köln; Mitglied, Bundesverband Auge e.V., Nürnberg; Sprecher der DFG Forschergruppe FOR 2240 (www.for2240.de); PI Horizon 2020 ARREST BLINDNESS; Action Chair EU COST BM1302 (www.biocornea.eu); Action Vice-Chair COST Aniridia (www.aniridia-net.eu); Herausgeber/Section Editor/ Beirat: Der Ophthalmologe, Klinische Monatsblätter für Augenheilkunde, CORNEA, Ophthalmic Research, Investigative Ophthalmology and Visual Sciences, Zeitschriftfür praktische Augenheilkunde; Gutachter:DFG, DOG, ERC, Belgian Research Fund, Dutch Ophthalmic Research Fund etc. Leitung, Task Force Research, Deutsche Ophthalmologische Gesellschaft DOG, München; Ehrenvorsitzender, Förderverein Forschung für das Sehen, Köln; Präsident/Vizepräsident der Deutschen Ophthalmologischen Gesellschaft DOG, München; Member, Steering Board, European Vision Institute, Brüssel, Belgien; Mitglied Berufsverband der Augenärzte Deutschlands e. V. (BVA); Mitglied Deutsche Ophthalmologische Gesellschaft (DOG); Mitglied The Association for Research in Vision and Ophthalmology (ARVO); Mitglied American Academy of Ophthalmology (AAO); Mitglied European Association for Vision and Eye Research (EVER); Mitglied Arbeitsgemeinschaft Deutscher Hornhautbanken; Mitglied The Cornea Society; Mitglied European Society of Cataract and Refractive Surgeons (ESCRS); Mitglied EuCornea.

\section{Literatur}

1. Flockerzi $E$, Maier $P$, Böhringer $D$, Reinshagen $H_{\text {, }}$ Kruse F, Cursiefen C, Reinhard T, Geerling G, Torun N, Seitz B, all German Keratoplasty Registry Contributors (2018) Trends in corneal transplantation from 2001 to 2016 in Germany: a report of the DOG-section cornea and its Keratoplasty registry. Am J Ophthalmol 188:91-98. https://doi.org/10.1016/j.ajo.2018.01.018

2. Matthaei M, Schrittenlocher S, Hos D, Siebelmann $S$, Bucher F, Schaub F, Hoerster R, Siggel R, Roters S, HeindILM, Bachmann B, Cursiefen C(2019) 10 years of Descemet membrane endothelial keratoplasty in Fuchs endothelial corneal dystrophy: what have we learned? Ophthalmologe 116(3):236-242

3. Enders P, Hall J, Bornhauser M, Mansouri K, Altay L, Schrader S, Dietlein TS, Bachmann BO, Neuhann T, Cursiefen C (2019) Telemetric intraocular pressure monitoring after boston keratoprosthesis surgery. Ophthalmology 126(2):322-324

4. Schaub F, Matthaei M, Enders P (2021) Versorgung von vaskularisierten Hochrisikoaugen mittels Boston Keratoprothese. Ophthalmologe. (In Press)

5. Hos D, Schlereth S, Schrittenlocher S et al (2021) DMEK in vaskularisierten Hochrisikoaugen und bei Transplantatversagen nach perforierender Keratoplastik. Ophthalmologe, in Press

6. Hayashi T, Zhang W, Hos D, Schrittenlocher S, Nhat Hung Le V, Siebelmann S, Matthaei M, Bock F, Bachmann B, Cursiefen C (2020) Descemet membrane endothelial keratoplasty in vascularized eyes: outcome and effect on corneal neovascularization. Cornea. https://doi.org/10.1097/ICO. 0000000000002502

7. Matthaei M, Hos D, Bock F et al (2021) Präkonditionierung von vaskularisierten Hochrisiko-Augen mittels Feinnadel-Diathermie und Crosslinking. Ophthalmologe, in Press

8. Hos D, Le VNH, Hellmich M, Siebelmann S, Roters S, Bachmann BO, Cursiefen C (2019) Risk of corneal graft rejection after high-risk keratoplasty following fine-needle vessel coagulation of corneal neovascularization combined with bevacizumab: a pilot study. Transplant Direct 5(5):e452-25 